\title{
An Online Survey of the Changing Trends of the Cardiac Surgeries in the Era of COVID-19
}

\author{
Virwar Kumar Jha \\ Department of cardiology, Jeevak Heart Hospital and Research Institute (P) Ltd., Patna, Bihar, India \\ ORCID: \\ Virwar Kumar Jha: https://orcid.org/0000-0003-2576-3955
}

\section{Abstract}

Objectives: To collect and assess cardiac surgeons' viewpoint about the changing cardiac surgery practices in India during COVID-19 pandemic. Methods: An online web-based study was conducted through an online questionnaire which was mailed to various cardiac surgeons in India (as retrieved from the cardiac surgeon directory). The responses were received and entered in an MS Excel spreadsheet and were analyzed. The ethical clearance for the study was not required as it was an online survey-based study without any patient data or treatment modification. Results: Out of 52 surgeons to whom the questionnaire was sent, 34 responded. Large number of surgeons (47.1\%) pointed that reduction in cardiac surgical volume for long time during pandemics is associated with surgical expertise attrition. Importantly, in addition to $44.1 \%$ response rate for substantial reduction of patient management in cardiac surgery during pandemic, $38.2 \%$ of surgeons responded for its complete discontinuation. Another $44.1 \%$ of surgeons responded that the risk of exposure in a COVID-19-negative subject during perioperative period is increased and affects outcomes. Multiple responses were obtained regarding repetition of COVID-19 testing in postoperative period. Conclusion: This pandemic is not going to end in the immediate future. However, to put cardiac surgery in standby mode till pandemic last is not an option. Measures should be taken by hospital and regulatory bodies to resume services of cardiac surgery in addition to containing ad preventing infection by COVID-19. In the absence of evidence-based recommendation, strong consensus opinion of practicing cardiac surgeon will guide clinical decision-making.

Keywords: Cardiac, COVID-19, surgery, questionnaire

\section{INTRODUCTION}

The clinical decision-making in cardiac surgery has never been affected to extent it is witnessed currently. Consequences of coronavirus disease 2019 pandemic is having significant implications on cardiac surgery both national and global. An internationally based quantitative survey has reported a median reduction in cardiac surgery case volume by $50 \%-75 \% .{ }^{[1]}$ We too have experienced this over the past 5 months. Besides infection by SARS-CoV 2 and its complications, other factors affecting cardiac surgery program are cost restrictions, manpower redistribution, and institutional reorganization to combat COVID-19 pandemic. ${ }^{[2]}$ Despite these constraints, salvage cardiac surgeries cannot be denied. Preexisting high-risk cardiac condition may be fatal if delayed. Thus, there is an uncertainty

Received: 20-11-2020 Revised: 10.01.2021 Accepted: 13-01-2021

Published Online: 29-03-2021

\begin{tabular}{|l|l|}
\hline \multicolumn{3}{|c|}{ Access this article online } \\
\hline Quick Response Code: & Website: \\
& \\
http://www.ijcva.com
\end{tabular}

regarding clinical decision-making in cardiac surgery. Due to this, there is variable clinical practice and patient outcome across cardiac units. In the absence of concrete evidence, consensus among surgeons will guide policy makers, clinicians, and patients. We conducted this survey with an aim to do collection and quantitative assessment of cardiac surgeons' view practicing in various regions of India during COVID-19 pandemic.

\section{Methods}

This was an observational cross-sectional study where we E-mailed a web-based questionnaire to a total of 52 consultant

Address for correspondence: Dr. Virwar Kumar Jha, Jeevak Heart Hospital and Research Institute (P) Ltd., Kankerbagh, Patna, Bihar, India.

E-mail: virwarjha@gmail.com

This is an open access journal, and articles are distributed under the terms of the Creative Commons Attribution-NonCommercial-ShareAlike 4.0 License, which allows others to remix, tweak, and build upon the work non-commercially, as long as appropriate credit is given and the new creations are licensed under the identical terms.

For reprints contact: WKHLRPMedknow_reprints@wolterskluwer.com

How to cite this article: Jha VK. An online survey of the changing trends of the cardiac surgeries in the era of COVID-19. Int J Cardiovasc Acad 2021;7:9-13. 
cardiac surgeons practicing in various regions of India on August 12, 2020. Since the study did not reveal any patient data or the personal details of any individual, no ethical clearance was obtained for the study.

All E-mail addresses of participants were retrieved from the Indian Association of Cardiothoracic Surgeon 2019 database. Our aim was to receive at least one response from each cardiac unit. In view of rapidly evolving circumstances, we predetermined to close the survey on August 22, 2020. This survey was primarily designed to gather information using 10 statements. Each statement was followed by participants' opinion on "which patient is considered for cardiac surgery under the current COVID-19 pandemic". Statistical results were expressed as percentage of responding consultant. Strong consensus was predefined as an opinion shared by at least $60 \%$ of responding consultants. ${ }^{[3]}$ The responses were received and entered into an MS Excel spreadsheet and were analyzed.

\section{Ethical statement}

Since the study did not reveal any patient data or the personal details of any individual, no ethical clearance was obtained for the study.

\section{RESULTS}

Of the total 1857 cardiac surgeons in India, the web-based questionnaire was sent successfully to 52 consultant cardiac surgeons practicing in various regions of India. Out of 52 consultants, 34 responded, which belonged to different states of the country as shown in Figure 1. Overall, the response rate was $65.4 \%$, which covered 19 cardiac units in various geographical regions of India as shown in Table 1. Maximum cardiac units were located in Delhi NCR. Table 2 illustrates the result of survey depicting the individual statement responses.

Analysis of survey data revealed that strong consensus (more than $60 \%$ of responses) was present for the following statements:

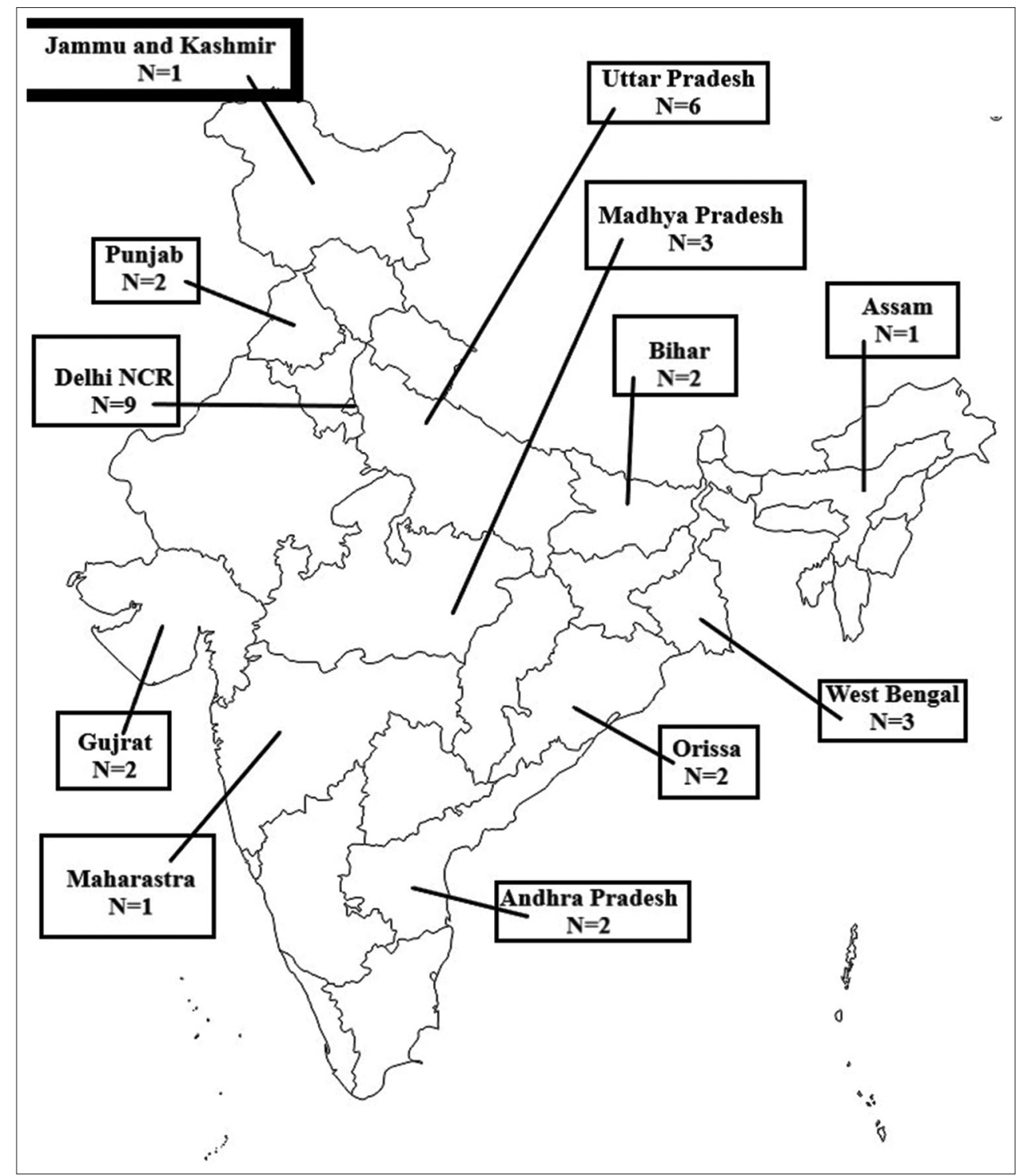

Figure 1: Number of responses from different states of the country 


\begin{tabular}{lc}
\hline $\begin{array}{l}\text { Table 1: Distribution of responding cardiac units of the } \\
\text { Country }\end{array}$ & Number of responding cardiac units \\
\hline State & 2 \\
\hline Andhra Pradesh & 1 \\
Assam & 1 \\
Bihar & 4 \\
Delhi NCR & 1 \\
Gujarat & 1 \\
Jammu and Kashmir & 2 \\
Madhya Pradesh & 1 \\
Maharashtra & 1 \\
Orissa & 1 \\
Punjab & 2 \\
Uttar Pradesh & 2 \\
West Bengal & \\
\hline
\end{tabular}

1. Preadmission COVID-19 screening test for elective cardiac surgery should be done for every case

2. Salvage cardiac surgery should be performed during pandemics with COVID-19 testing done before procedure

3. All members of cardiac team should adopt personal protective equipment (PPE) in every case regardless of COVID-19 status.

For rest of the statements, there was no strong consensus; however, still more than $50 \%$ response was present for,

1. Fund diversion and resource relocation during pandemics has compromised cardiac surgery program to variable extent

2. Elective cardiac surgery in COVID-19 subject should be postponed till the patient is declared COVID-19 negative and fit for surgery.

Large number of surgeons (47.1\%) pointed that reduction in cardiac surgical volume for long time during pandemics is associated with surgical expertise attrition. Importantly, in addition to $44.1 \%$ response rate for substantial reduction of patient management in cardiac surgery during pandemic, $38.2 \%$ of surgeons responded for its complete discontinuation. Another $44.1 \%$ of surgeons responded that the risk of exposure in a COVID-19-negative subject during perioperative period is increased and affects outcomes. Multiple responses were obtained regarding repetition of COVID-19 testing in postoperative period.

\section{DISCUSSION}

Even after extensive search in the history of cardiac surgery, no report of any pandemic interference was found. As the COVID-19 pandemic is evolving across the globe, advisories have been published regarding patient management in cardiac surgery. ${ }^{[4]}$ Country has been compelled to implement strict measures to contain rapid transmissibility of COVID-19. This has led to postponement of elective cardiac surgical cases. Cardiac surgical programs have been restricted to triage of emergency and urgent cases only. Reduction in pediatric cardiac surgical output to $10 \%$ of normal output has been reported. ${ }^{[5]}$ Surgical case load of cardiothoracic surgery department at a tertiary center has been reduced to about $2 \%-4 \%$ of its normal. ${ }^{[6]}$ It is by necessity that resources have been diverted away from cardiac surgery, and in many instances, cardiac units have been repurposed as COVID-19 units. Shortages of PPE, sanitation products, ventilators, and intensive care unit beds have been widely reported. In this study also, $38.2 \%$ of participants responded for complete discontinuation and other $44.1 \%$ for substantial reduction in cardiac surgical patient management. One concern associated with the results is the underutilization of practice and loss of skill by highly trained personnel. ${ }^{[5]}$

Some of the challenges imposed by current COVID-19 pandemic are accuracy of diagnostic tests; waning of immunity; possible reinfection; and possibility that degree of social distancing might change with time and lack of definitive, preventive, and therapeutic measures. Moreover, continuously changing dynamics of current pandemic makes future unpredictable. Therefore, evidence for safety of cardiac surgery, risk of COVID-19 exposure and infection in hospital setting, and influence of diversion of personnel and resources away from cardiac surgery is not known. In this respect, as seen in the present study, 58.8\% response for compromised cardiac surgery program (due to resource redistribution) and $44.1 \%$ for increased risk of exposure have affected the outcomes, whereby it gives an impression of the extent to which COVID-19 pandemic has impacted cardiac surgery program across the population.

We have been reported by patients or their kin about their reluctance to attend OPD due to lack of transportation facilities and risk of exposure to COVID-19. Unpredictable status of perioperative COVID-19 exposure and consequent mortality, together with lack of risk stratification model, predisposes patients to COVID-19 exposure and makes their counseling challenging. For these reasons, patient consent for cardiac surgery is delayed. ${ }^{[7]}$ In the absence of data-driven evidence result, no definitive recommendation is available for case selection and clinical decision-making. However, as the pandemic is progressing, clinical observation is accumulating slowly. In this survey, on the one hand, $47.1 \%$ of participants accepted that recommendation has remained unimpacted, and on the other hand, $29.4 \%$ agreed that recommendation has been impacted and other $23.5 \%$ accepted for not knowing. Relevant results of this survey are consistent with published advisories. ${ }^{[4,7]}$

In our study, $91.2 \%$ of the cardiac surgeons agreed that prior to elective cardiac surgery, COVID-19 screening should be done. With regard to repetition of COVID-19 testing in postoperative period, results are variable, still largest figure of $47.1 \%$ agreed for repeat COVID-19 testing in every case irrespective of COVID-19 testing. In addition to contact history, travel history and typical sign symptoms options available for screening test are nasopharyngeal swab, high- 


\section{Table 2: Distribution of responses to the survey}

During this pandemic, patient management in cardiac surgery has been affected

a) Only marginally
b) Substantially reduced
c) Completely discontinued

Fund diversion and resource relocation during pandemics has compromised cardiac surgery program

a) $\mathrm{No}$

b) Yes to variable extent $20(58.82)$

c) Cannot comment

$7(20.59)$

COVID-19 pandemic had impacted recommendations in cardiac surgery
a) I don't know
$8(23.53)$
b) Yes
$10(29.41)$
c) $\mathrm{No}$
$16(47.06)$

Preadmission COVID-19 screening test for elective cardiac surgery should be done

b) For suspected case only

c) For every case

Salvage cardiac surgery should be performed during pandemics

a) I don't know
b) Yes, with COVID-19 testing before procedure
c) Yes, without COVID-19 testing before procedure

Elective cardiac surgery in a COVID-19 subject should be scheduled

c) Postponed till patient is declared COVID-19 negative and fit for surgery

All members of cardiac team should adopt PPE

a) In COVID-19 confirmed case only

b) In every case regardless of COVID-19 status

Risk of exposure in a COVID-19 negative subject during perioperative period

a) I don't Know

b) Increased and affect outcome

c) Increased but does not affect out come

d) No risk

COVID-19 testing should be repeated in postoperative period

a) I don't know

b) Every case irrespective of preoperative status

resolution computed tomography chest, and immunological serum test. Although statement has been kept simple, it is important that status of each option or its combination must be recognized. In the resource-limited setting, pre-test risk stratification based on patient symptom, exposure, and contact history and community prevalence is helpful because in low prevalence setting, positive predictive value is low and in high prevalence setting negative predictive value is low. ${ }^{[8]}$ The initial and most common test is the reverse transcription-polymerase chain reaction of the 1aboratory region of the SARS-CoV-2 virus. Although there may be significant variability in result between laboratories. A rapid immunoassay detecting IgG and IgM antibody to SARSCOV 2 is also available. However, neither a reactive antibody test be sole basis for diagnosis of disease nor a negative test rue out the SARS COV2 infection.

Similar to the result obtained by Benedetto et al.,${ }^{[7]} 64.7 \%$ of surgeons accepted that PPE kit should be adopted by all members of cardiac team irrespective of COVID-19 status of patient. Different levels of PPE for different members of cardiac team have become available. ${ }^{[4]}$ The risk of exposure and infection to cardiac surgeons is unknown. Obvious sources of exposure include aerosol from ventilators, 
respiratory treatments, chest tube air leaks, and electrocautery. Reoperations are particularly risky because of the potential for parenchymal lung injury during sternal reentry and mediastinal dissection.

A dynamic triage plan has been proposed based on local and regional COVID-19 prevalence. High acuity cases, namely acute aortic dissection of ascending aorta, significant left amin stenosis with unstable ischemia symptom, acute ischemic mitral regurgitation with unstable ischemic symptoms, and others, should not be deferred. For these cases, one can proceed with high-risk lifesaving surgery provided COVID-19 PPE and precaution. With COVID-19 PPE, precaution, and critical illness not attributable to COVID-19, high-risk lifesaving surgery is advisable. ${ }^{[8]}$ In this survey also, $64.7 \%$ of the surgeons agreed that salvage cardiac surgery should be performed with COVID-19 testing before procedure. Even in emergency cases if critical illness id attributable COVID-19, surgery should be avoided for futility and the ethical committee review should be obtained. Elective low acuity cases should be deferred beyond 12 weeks in addition majority (55.9\%) agreed that in a COVID-positive subject even such elective cases should be postponed till the patient is declared COVID-negative and fit for surgery.

\section{Conclusion}

This pandemic is not going to end in the immediate future. Therefore, to put cardiac surgery in standby mode till pandemic last is not an option. Measures should be taken by hospital and regulatory bodies to resume services of cardiac surgery in addition to containing and preventing infection by COVID-19. In the absence of evidence-based recommendation, strong consensus opinion of practicing cardiac surgeon will guide clinical decision-making. We are facing a devastating viral pandemic for which we were unprepared. Viral pandemic has surfaced the earth often, so health-care system should be having policies such that any such event in future does not compromise other services and also save life of millions of human being.

\section{Financial support and sponsorship}

Nil.

\section{Conflicts of interest}

There are no conflicts of interest.

\section{References}

1. Gaudino M, Chikwe J, Hameed I, Robinson NB, Fremes SE, Ruel M. Response of cardiac surgery units to COVID-19: An internationally-based quantitative survey. Circulation 2020;142:300-2.

2. Gopal K, Varma PK. Cardiac surgery during the times of COVID-19. Indian J Thorac Cardiovasc Surg 2020;20:1-2.

3. Kea B, Sun BC. Consensus development for healthcare professionals. Intern Emerg Med 2015;10:373-83.

4. Hiremath CS, Yadava OP, MeharwalZS, IyerKS, Velayudhan B, COVID-19 Task Force of the Indian Association of Cardiovascular-Thoracic Surgeons. IACTS guidelines: Practice of cardiovascular and thoracic surgery in the COVID-19 era. Indian J Thorac Cardiovasc Surg 2020;36:1-3.

5. Iyer KS. Impact of the COVID-19 pandemic on pediatric cardiac care in India: Time for action! Ann Pediatr Card 2020;13:183-5.

6. Hote M, Gupta SS. Cardiothoracic surgery practice at a tertiary center during the COVID-19 pandemic. J Pract Cardiovasc Sci 2020;6:105-7.

7. Benedetto U, Goodwin A, Kendall S, Uppal R, Akowuah E. A nationwide survey of UK cardiac surgeons' view on clinical decision making during the coronavirus disease 2019 (COVID-19) pandemic. J Thorac Cardiovasc Surg 2020;160:968-73.

8. Patel V, Jimenez E, Cornwell L, Tran T, Paniagua D, Denktas AE, et al. Cardiac surgery during the COVID-19 pandemic: Perioperative considerations and triage recommendations. J Am Heart Assoc 2020;9:e17042. 